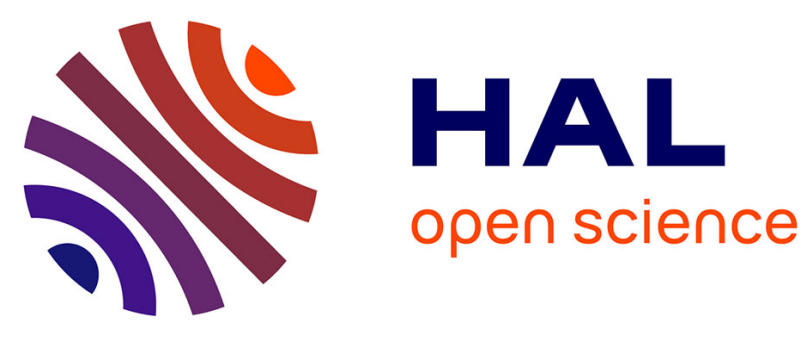

\title{
Full field modeling of recrystallization: Effect of intragranular strain gradients on grain boundary shape and kinetics
}

Dmitrii Nikolaevich Ilin, Nathalie Bozzolo, Thomas Toulorge, Marc Bernacki

\section{- To cite this version:}

Dmitrii Nikolaevich Ilin, Nathalie Bozzolo, Thomas Toulorge, Marc Bernacki. Full field modeling of recrystallization: Effect of intragranular strain gradients on grain boundary shape and kinetics. Computational Materials Science, 2018, 150, pp.149 - 161. 10.1016/j.commatsci.2018.03.063 . hal01770044

HAL Id: hal-01770044

https://hal-mines-paristech.archives-ouvertes.fr/hal-01770044

Submitted on 4 May 2018

HAL is a multi-disciplinary open access archive for the deposit and dissemination of scientific research documents, whether they are published or not. The documents may come from teaching and research institutions in France or abroad, or from public or private research centers.
L'archive ouverte pluridisciplinaire HAL, est destinée au dépôt et à la diffusion de documents scientifiques de niveau recherche, publiés ou non, émanant des établissements d'enseignement et de recherche français ou étrangers, des laboratoires publics ou privés. 


\title{
Full field modeling of recrystallization: effect of intragranular strain gradients on grain boundary shape and kinetics
}

\author{
D. N. Ilin, N. Bozzolo, T. Toulorge, M. Bernacki* \\ Mines-ParisTech, PSL-Research University, CEMEF Centre de mise en forme des matériaux, CNRS UMR \\ 7635, CS 10207 rue Claude Daunesse, 06904 Sophia Antipolis Cedex, France
}

\begin{abstract}
The effect of stored energy field heterogeneity on the microstructure evolution during static recrystallization (SRX) was assessed by performing three dimensional (3D) full field simulations. These simulations were performed by using the Level set (LS) method in a finite element (FE) framework with unstructured tetrahedral FE mesh. An extension of classical LS-FE for SRX was developed to account for average stored energies in zones adjacent to grain boundaries (GBs) in each grain. Annihilation of dislocations by a passing GB was taken into account. The results of 3D simulations were analyzed to compare the "per interface" LS model to the usual "per grain" LS method (with averaging stored energy per grain). Recrystallization is faster at the beginning of the heat treatment when using the "per interface" model. The analysis of the evolution of grain volume and grain surface scattering reveals that the "per interface" approach results in complex shapes of grains which are closer to those observed in experiments. Local simulations for few grains with refined FE mesh were also performed to study the effect of stored energy heterogeneity along interfaces, and even higher complexity of grain topology was observed compared to the results of "per interface" computations.
\end{abstract}

Keywords: Recrystallization, grain boundary mean curvature, Intragranular stored energy gradients, FE simulation, Level Set.

\footnotetext{
* Corresponding author

Email address: marc. bernacki@mines-paristech.fr (M. Bernacki)
} 


\section{Introduction}

Understanding of microstructure evolution during thermomechanical processing is crucial for the prediction and optimization of mechanical properties of metals and alloys. Numerical simulations can contribute to the better description of static, dynamic and postdynamic recrystallization, grain growth and Smith-Zener pinning phenomenon [1]. In a full field context, they can be performed using probabilistic Monte Carlo Potts [2-6], Celullar Automata [7,-9], deterministic phase field [10, 12], vertex [13, 14] or level set (LS) [15-26] models. These numerical methods are currently used and developed by numerous researchers [27] and regularly compared for particular metallurgical mechanisms [13, 19].

Of course, all the mentioned models have their own strengths and weaknesses. The LS method, with recent developments of optimization techniques, namely, a direct and parallel reinitialization algorithm [28] and a recoloring scheme [29] coupled with a meshing/remeshing strategy in context of unstructured FE mesh can be considered as an efficient and accurate way to model large systems evolution for static [20] or dynamic recrystallization [21]. However, many challenges remain for modeling microstructure evolution with adequate description of all physical aspects at the microscopic scale and in 3D. Influences of stored energy heterogeneities at the grain scale and anisotropy of mobility and interface energy (by taken into account the entire five dimensional domain of GB existence in the description of the GB energy) need to be further investigated. To take into account these effects in the FE-LS approach, a careful analysis of the solution behavior is required since instabilities can raise from heterogeneous velocity field [17].

In previous works using the LS method for modeling the Smith-Zener pinning phenomenon or recrystallization, the effect of dislocation density heterogeneities on the GBs motion was taken into account at the mesoscopic scale by averaging the stored energy per grain (and computing the corresponding energy gradient across GBs). To the authors knowledge, this approximation is systematic for the deterministic methods except in the full field formulation of [30] where, however, the heterogeneous intragran- 
ular stored energy field and its evolution are not taken into account directly. The "per grain" approach provides quite good agreement of computed recrystallization kinetics with experimental ones but GBs keep regular shapes without remarkable development of curvatures [20, 22-24]. Similar grain topology can be found in a recent paper reporting the results of the vertex method coupled with a crystal plasticity model [31]. Results of simulations without averaging procedure using the Monte Carlo Potts model [3-6] or Celullar Automata [7-9] reported in the literature do not put in evidence either the development of irregular shapes of GBs at the transient states of recrystallized microstructures. The experimental measurements, however, often reveal the appearance of distorted GBs during recrystallization as illustrated by the white arrows in Fig 1 for an experimental micrograph obtained with the Forward Scattered Electrons (FSE) detector of a Scanning Electron Microscope (SEM) on a partially recrystallized tantalum sample.

In the present work, the effect of stored energy heterogeneity was assessed at the intragranular scale by considering the stored energy per each interface (GB) of a given grain. The energies on one side of each GBs and on its other side can differ due to the heterogeneity of the original stored energy field, as provided by crystal plasticity FE computations [26, 32], thereby establishing an energy gradient which contributes to the GBs motion. This approach still provides an approximate estimation of recrystallization kinetics but more accurately from the physical point of view than "per grain" method [20, 21], notably at the multiple junctions. In addition, in the developed "per interface" approach, a minimal $e_{0}$ value (material dependant) for the stored energy energy is imposed, as in [21] for "per grain method", in recrystallized zones to account for the annihilation of dislocations by a passing GB. The stored energies near interfaces are updated at each time step. The GB energy and mobility were assumed to be isotropic in the following.

The proposed method was first validated considering simple configurations. Namely, the simulations of 2D and 3D three grain junction motion with constant velocities were performed, and the results were compared with analytical solution discussed in the literature [33]. Then, 3D simulations of static recrystallization were carried out and compared with ones obtained in [20] for 304L stainless steel. Finally, the numerical 
study was conducted on few grains with refined mesh without averaging to analyze the effect of stored energy heterogeneities along GB. The results were compared with ones obtained using the "per interface" approach.

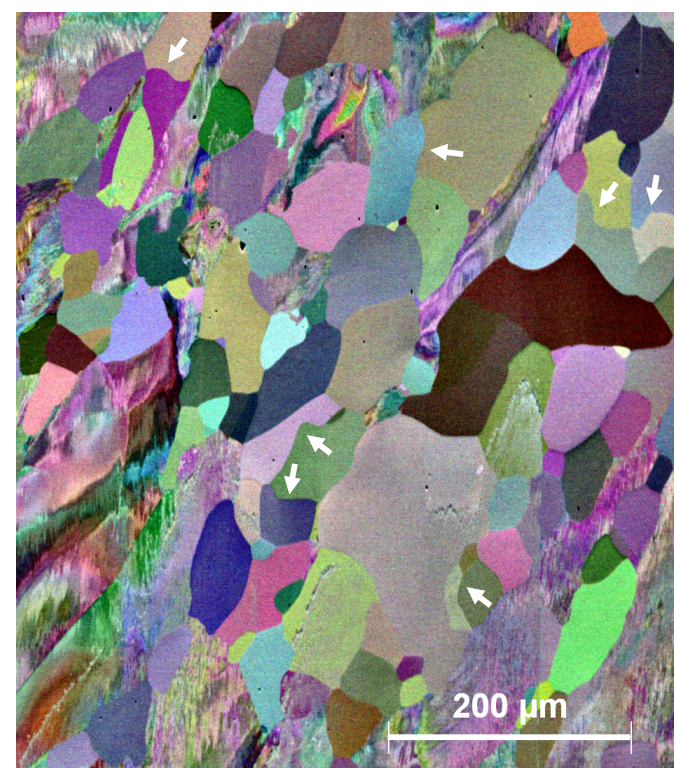

Figure 1: Experimental micrograph obtained with the FSE detector of a SEM on a partially recrystallized tantalum sample. The color contrast is orientation dependent. Recrystallized grains appears with an homogeneous color contrary to the unrecrystallized ones exhibiting substructures. White arrows indicate few examples of grain boundaries between recrystallized grains with a complex shape.

\section{Numerical model}

\subsection{Level set description of the polycrystals}

In this work, the full field modeling was performed by using the LS method in a P1 FE framework. In the LS approach, each sub-domain $G$ (grain) in a given domain $\Omega$ (polycrystal) is classicaly described implicitely by computing the signed distance function $\phi(x, t)$ representing the distance to the sub-domain boundaries $\Gamma=\partial G$ (grain boundaries). In the used P1 formulation, the function $\phi(x, t)$ is calculated at each node on the FE mesh and is chosen, by convention, positive inside of the grain and negative 
outside:

$$
\left\{\begin{array}{l}
\phi(x, t)= \pm d(x, \Gamma(t)), x \in \Omega \\
\Gamma(t)=\{x \in \Omega: \phi(x, t)=0\}
\end{array}\right.
$$

where $d(x, \Gamma(t))$ means the Euclidean distance from the point $x \in \Omega$ to the boundary $\Gamma(t)$. In the LS method, the evolution of $\phi(x, t)$ is given by the following transport equation [15]:

$$
\left\{\begin{array}{l}
\frac{\partial \phi(x, t)}{\partial t}+\vec{v}(x, t) \cdot \nabla \phi(x, t)=0, \\
\phi(x, t=0)=\phi^{0}(x)
\end{array}\right.
$$

where $\vec{v}(x, t)$ is the velocity field. It is generally assumed for metals that the kinetic law for grain boundary motion can be defined as [34]:

$$
\vec{v}=M P \vec{n},
$$

where $M$ is the grain boundary mobility, $P$ is the net pressure i.e. the net driving force per unit area, and $\vec{n}$ is the outward unit normal to the GB. In context of deterministic full field approaches, the net pressure is classically defined as:

$$
P=\tau \llbracket \rho \rrbracket-\gamma \kappa,
$$

where $\tau$ is the dislocation line energy, $\llbracket \rho \rrbracket$ is the dislocation density jump across interfaces, $\gamma$ is the GB energy and $\kappa$ is the mean GB curvature (i.e. the curvature in $2 \mathrm{D}$ and the sum of main curvatures in 3D). That is the GB motion is governed by the stored energy gradient across the GBs and capillarity. The isotropy hypothesis remains here to consider $M$ as only dependant of the temperature and $\gamma$ as constant. Let's consider a polycrystal consisting of $N_{g}$ grains and $N_{p}$ LS functions are used to represent the polycrystalline aggregate. Assuming that $\left\|\nabla \phi_{i}(x, t)\right\|=1, \forall i \in \llbracket 1, N_{p} \rrbracket$, i.e. $\phi_{i}$ remain distance functions all along the simulation, after substituting Eq.(4) into Eq.(3) and Eq. (3) into Eq.(2), and by considering the following properties of distance functions with the chosen sign convention,

$$
\vec{n}_{i}=-\nabla \phi_{i} /\left\|\nabla \phi_{i}\right\|=-\nabla \phi_{i}, \quad \kappa_{i}=\nabla \cdot \vec{n}_{i}=-\Delta \phi_{i},
$$


, one can solve a set of $N_{p}$ convective-diffusive equations to take into account Eq.33 for all the grains of the considered polycrystal [18]:

$$
\left\{\begin{array}{l}
\frac{\partial \phi_{i}(x, t)}{\partial t}-M \gamma \Delta \phi_{i}(x, t)+\vec{v}_{i}^{\llbracket \rho \rrbracket} \cdot \nabla \phi_{i}(x, t)=0, \\
\vec{v}_{i}^{\llbracket \rho \rrbracket}=M \tau \llbracket \rho \rrbracket_{i} \vec{n}_{i}, \\
\phi_{i}(x, t=0)=\phi_{i}^{0}(x) .
\end{array}\right.
$$

Details concerning how $\vec{v}_{i}^{\llbracket \rho \rrbracket}$ is finally decomposed by considering a "per grain" or a "per interface" approach are described in the following sections. In the case where $N_{p}=N_{g}$, the number of equations is equal to the number of grains. In the present work, we use the coloring scheme [29] which allows to limit the number of needed LS functions. The polycrystal is decomposed into families of distinct grains (not neighboring), and each family is represented by a single Global Level set (GLS) function $\phi_{i}(x, t)$ verifying Eq 6. The recoloring scheme introduced in [29] and improved in [20] is used: each family contains only the grains which are separated by a critical distance. To avoid the numerical coalescence of grains represented by the same GLS function, a dynamic swapping procedure (recoloring) is performed at each time step (see [20, 29] for details). This aspect is crucial in terms of memory and numerical cost to brave 3D LS-FE simulations with a large number of grains.

A particular numerical treatment was proposed in [35] to avoid kinematic incompatibilities after solving the convective-diffusive equations (6). The method consists in removing vacuum regions appearing at multiple junctions after each time increment by correcting the GLS functions as follows:

$$
\phi_{i}(x, t)=\frac{1}{2}\left(\phi_{i}(x, t)-\max _{j \neq i} \phi_{j}(x, t)\right), 1 \leq i \leq N_{p} .
$$

One of the drawbacks of LS method is a possible alteration of the distance metric after solving Eq. 6 : $:\left\|\phi_{i}(x, t)\right\| \neq 1$. The distance function must therefore be reinitialized at each time step to ensure the metric property $\left\|\phi_{i}(x, t)\right\|=1$ and, hence, the validity of the convective-diffusive formulation Eq. (6) at least in a narrow band around the grain interfaces $\left(\left\|\phi_{i}(x, t)\right\|<h\right)$. It is performed by using the parallel and direct reinitialization algorithm detailed in [28]. This method was demonstrated to be fast and accurate. In the reinitialization algorithm, the GLS interface is firstly discretized into a collection 
of segments. Nodal values of the GLS functions are then recomputed by finding the nearest element of the collection and calculating the distance between the considered node and this nearest element. This method takes advantage of a space-partitioning technique using $k$ - $d$ tree and an efficient bounding box strategy enabling to maximize the numerical efficiency for parallel computations.

\subsection{Grain boundary motion - classical LS formulation}

By extrapolate the shape of the velocity term, $\vec{v}_{i}^{[\rho \rrbracket}$ of Eq. $[6$, for the interface between grain $G_{i}$ and $G_{j}$, the corresponding velocity, $\vec{v}_{i j}^{\llbracket \rho \rrbracket}$, can be written as:

$$
\vec{v}_{i j}^{\llbracket \rho \rrbracket}=M \tau \llbracket \rho \rrbracket_{i j} \vec{n}_{i j},
$$

In the usual approach considering the stored energy as constant per grain [17, 18 , 20- 23, 26, 32], for each interface between grain $G_{i}$ and $G_{j}$ it is assumed that

$$
\tau \llbracket \rho \rrbracket_{i j}=e_{j}-e_{i},
$$

where $e_{i}$ and $e_{j}$ are the mean stored energies in the grains $G_{i}$ and $G_{j}$, respectively. These averages can directly come from constant approximative values where only a gradient of the stored dislocations between the nucleus and the non-recrystallized grains is considered as in [17, 18, 23], simplified mechanical formulations as in [21] or be evaluated thanks to an existing dislocation field in the FE mesh of the calculation domain $\Omega$ following Eq. 10 as in [20, 22, 26, 32].

$$
e_{i}=\tau \int_{G_{i}} \rho(x) \mathrm{d} \Omega / \int_{G_{i}} \mathrm{~d} \Omega .
$$

In the present work, to model the recrystallization kinetics with higher accuracy from the physical point of view, we develop an approach in which the rate of interface migration is computed locally by averaging the stored energy per interface, namely, over the nodes of the FE mesh which are closer to a given interface than to the others. For this averaging procedure, (9) can be rewritten as follows:

$$
\tau \llbracket \rho \rrbracket_{i j}=e_{j i}-e_{i j}
$$


where $e_{i j}$ and $e_{j i}$ are an average of the stored energy field at interface $\Gamma_{i j}$ in the grains $G_{i}$ and $G_{j}$, respectively, as defined by Eq. 12 and illustrated in Fig. 2 .

$$
e_{i j}=\tau \int_{\Gamma_{i j}} \rho(x) \mathrm{d} \Omega / \int_{\Gamma_{i j}} \mathrm{~d} \Omega
$$

with,

$$
\Gamma_{i j}=\left\{x \in \Omega / \phi_{i}(x, t) \in[0, h], \text { and } \phi_{j}(x, t)=\max _{k \neq i}\left(\phi_{k}(x, t)\right)\right\}
$$

Thus, when computing the stored energy per interface, we consider only the nodes for which the distance from the interface is less than the parameter $h$ (half thickness of the interface zone presented in Fig. 2 chosen by the user. The value of $h$ should be imposed taking into account the mesh size. The details of the method to identify the interfaces and to compute the corresponding energies are given in the subsection 2.3

\section{3. "Per interface" method}

The convection-diffusion equations (Eq 6 under zero Neumann boundary condition (implying the orthogonality of the grain boundary network to to the calculation domain border) are solved in the following by using an implicit streamline upwind Petrov-Galerkin FE scheme. Using this numerical scheme, a special attention has to be paid to the velocity field $\vec{v}^{\llbracket \rho \rrbracket}$ in the vicinity of multiple junctions as it was emphasized

in [17]. In fact, rather than to deal with $\vec{v}_{i}^{[\rho \rrbracket}$ per grain as described in Eq. 6) and by considering the contributions of each neighbor as detailed by Eq.(8), a global common velocity, $\vec{v}_{e}$, is built in the calculation domain and used for each convection-diffusion system. For the "per interface" approach developed in the present work, the following smoothing is naturally proposed as an extension of the method formulated in [17] for the "per grain" approach:

$$
\forall x: 0 \leq \phi_{i}(x, t)<h \quad \vec{v}_{e}(x, t)=M \sum_{\Gamma_{i j}} f\left(\phi_{i}(x, t), l\right)\left(e_{i j}(x, t)-e_{j i}(x, t)\right) \vec{n}_{i j}(x, t),
$$

The function $f$ must be chosen as a monotonic function decreasing from 1 (for $\phi_{i}=0$ ) to 0 (for $\phi_{i}=l$ ). 


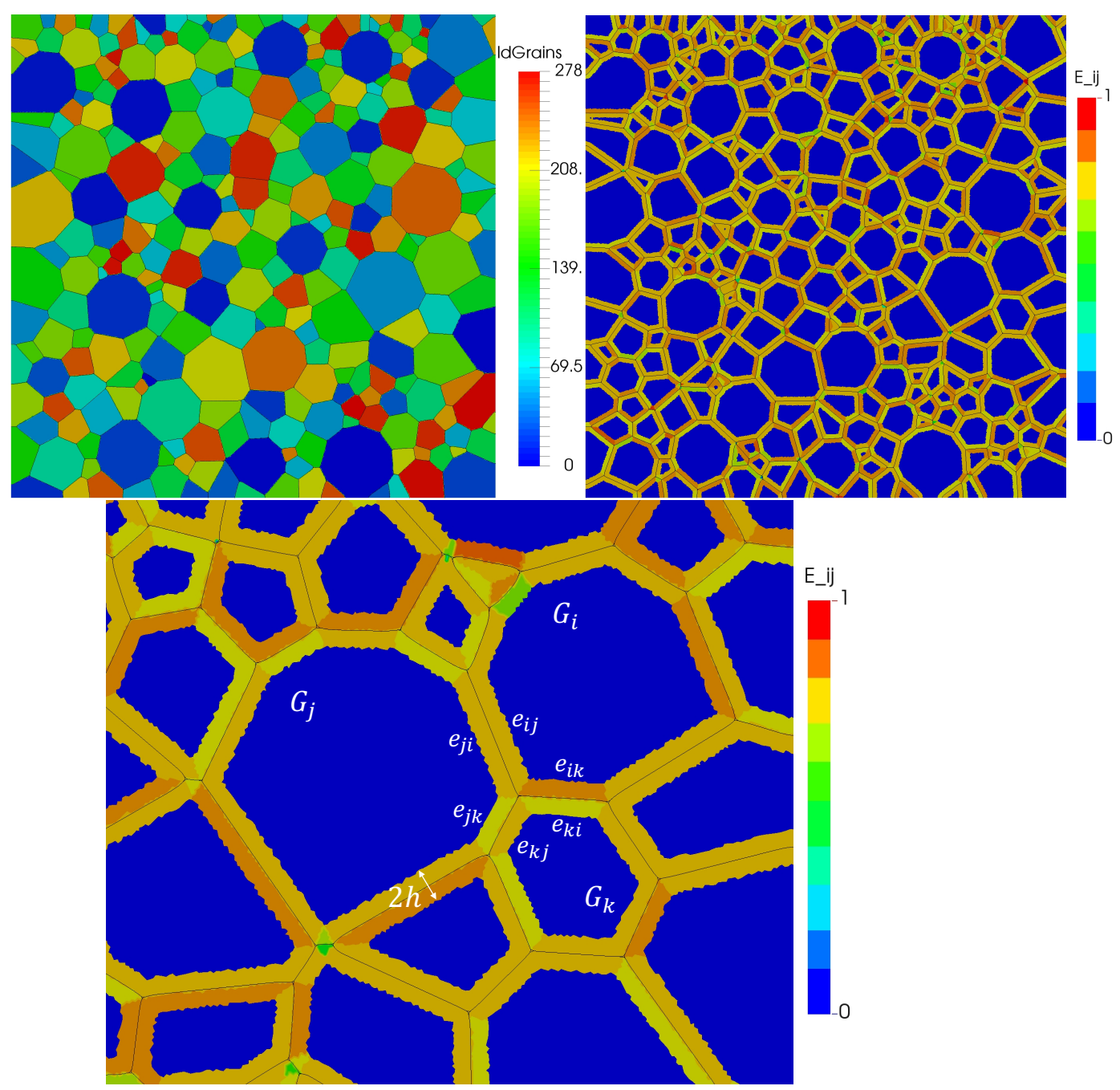

Figure 2: 2D example of dimensionless stored energy field averaged per interface zone of thickness $h$ for a polycrystal made of 278 grains and generated thanks to a Laguerre-Voronoï tessellation algorithm: (top left) polycrystal with grains'Id, (top right) averaged stored energy field and (bottom) zoom on few grains. 
To identify interfaces and to compute the corresponding stored energy difference $e_{i j}-e_{j i}$ in [14], four indices need to be recorded for all the nodes located in the vicinity of the interfaces:

1) index of the grain family, $i$;

2 ) index number of the grain, $I_{i}$ given by the local value of the index field $I_{i}(x, t)$;

3 ) index of the neighboring grain family, $j$;

4) index number of the neighboring grain, $I_{j}$ given by the local value of the index field $I_{j}(x, t)$;

First, for each GLS function $\phi_{i}$, the corresponding index field is defined in the nodes of the FE mesh. Inside of a grain the field is equal to the grain index which is the natural number from 1 to $N_{G}^{i}$ where $N_{G}^{i}$ is the total number of grains of the corresponding family. Fig 3 (top) illustrates, for the polycrystal of Fig 2 one of the index field, $I_{3}(x, t)$, obtained after the coloring algorithm whith $N_{G}^{i}=45$. Nine GLS are used in this case to describe all the microstructure. The index field is equal to 0 in the nodes which are outside of the grains belonging to the corresponding GLS function, i.e. $\phi_{3}$. The reader is referred to [20, 29] for details.

Let's assume that the grain $G$ in Fig. 3 (bottom) belongs to one of the grain families represented by GLS function $\phi_{i}$ and then that the index field inside of this grain is equal to $I_{i}$. In each node of the mesh with coordinates $x$ located inside of the grain $G$ and close to one interface of this grain (i.e. $0 \leq \phi_{i}(x, t)<h$ ), a neighboring (the nearest) grain family is identified (family with maximal value of the corresponding GLS function, $\left.\phi_{j}(x, t)\right)$. The index of this neighboring family, $j$, is recorded. These three indices $\left(i, I_{i}\right.$ and $j$ ) are sufficient to uniquely identify the interface only if the grain $G$ does not have several neighboring grains which belong to the same grain family. In the opposite case, the fourth index is required.

It is exactly the case of the grains 13, 20 and 35 in Fig. 3 (bottom) which all belong to the same GLS, i.e. $I_{3}$. To distinguish the interfaces between the grain $G$ and these grains the fourth index, $I_{3}$, is then required to obtain the index number of these grains (13, 20 and 35). The index field for the the third grain family is extended as it is illustrated in Fig. 3 (bottom) up to the distance $h$ from the boundaries of the grains which allows to define the index number of neighboring grains $I_{3}$ in nodes located in 


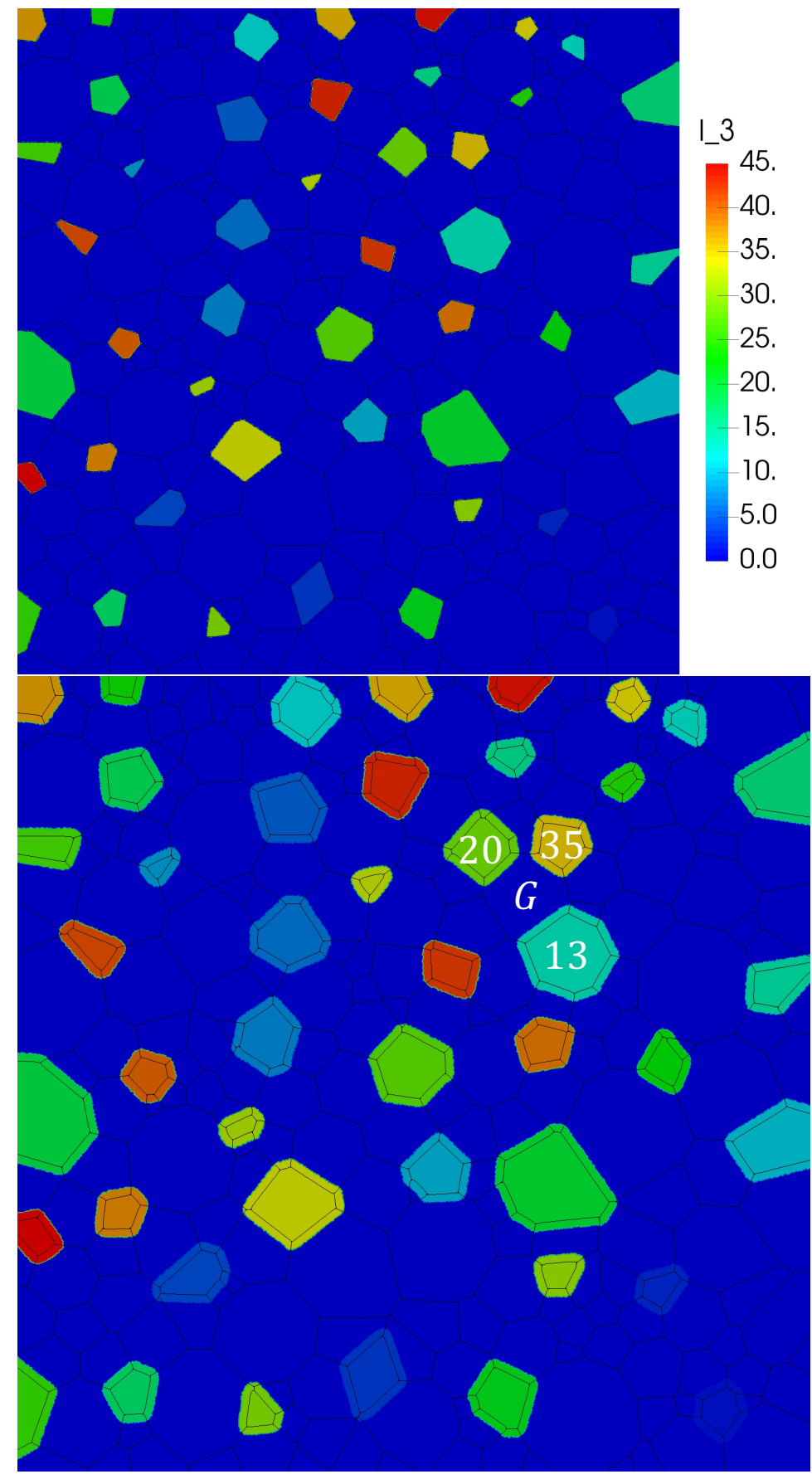

Figure 3: Description of the $I_{3}(x, t)$ index field for the polycrystal described in Fig 2 (top) and description of one particular configuration requiring the use of the fourth index (bottom). 
the grain $G$ close to the interfaces (i.e. $\left.0 \leq \phi_{i}(x, t)<h\right)$.

After performing, for all grain families, the procedure described above, the averaged stored energy per interface $e[i]\left[I_{i}\right][j]\left[I_{j}\right]$ can be computed. Fig. 2 illustrates an example of the stored energy field averaged per interface.

The energy difference across the interface can then be easily then calculated in Eq. 14 as:

$$
e_{i j}(x, t)-e_{j i}(x, t)=e[i]\left[I_{i}\right][j]\left[I_{j}\right]-e[j]\left[I_{j}\right][i]\left[I_{i}\right] .
$$

An alternative to the proposed four index algorithm could be to consider a fixed index for each grain from appearance to disappearance of it independently of the coloring and recoloring operations defining the global LS functions.

In the present work, the minimal $e_{0}$ value (material dependant) for the stored energy is imposed in recrystallized zones to account for the annihilation of dislocations by a passing GBs [36]. The energy per interface is recomputed after each time increment.

\section{Discussions on simple configurations}

\subsection{Three grain junction motion: dependent velocities}

Let's consider a simple isotropic (in terms of $M \gamma$ value) stable triple junction configuration described in Fig. 4 Initially, the interfaces meet at the angles of 120 degrees which correspond, following Herring's equation, to the equilibrium state for the angles in the case of isotropic grain boundary energy. Following Eq. 6 by assuming a constant stored energy per grain with $e_{1}>e_{2}>e_{3}$, the velocities of the straight interfaces (see Fig. 4(a)) are defined by:

$$
\left\|v_{13}\right\|=M \tau\left(e_{1}-e_{3}\right), \quad\left\|v_{23}\right\|=M \tau\left(e_{2}-e_{3}\right),
$$

and,

$$
\left\|v_{12}\right\|=M \tau\left(e_{1}-e_{2}\right)=\left\|v_{13}\right\|-\left\|v_{23}\right\| .
$$

It can be be easily shown that the condition (17) automatically yields the transport of the initial configuration by conserving the angles of 120 degrees without boundary 
distortion as illustrated by the dotted lines in Fig. 4(a). Indeed, the following statement can be formulated:

- if three lines intersect at a single point at angles of 120 degrees, and if one of the lines is displaced in its perpendicular direction to the distance of $a>0$ and one of the two others is moved to the distance of $b>0$ from the initial positions, then the distance between the intersection point for the displaced lines and the third (not moved) line is equal to $|a-b|$ or to $a+b$ (depending on the direction of lines displacement). The proof of this statement is quite obvious and based on simple geometrical rules.

Thus, when the "per grain" approach is employed for isotropic configuration, the velocities are compatibles in the sense that they are dependent and the displacement of interfaces does not disturb the geometry of the triple junctions initially considering at the equilibrium state.

However, the interface distortion can appear if the velocities are independent which is, a priori, the case when using the "per interface" approach. This can be understood by analyzing an example of triple junction motion with incompatible velocities illustrated in Fig. $4 \mathrm{~b}$ where $\left\|v_{13}\right\|=M \tau\left(e_{13}-e_{31}\right),\left\|v_{23}\right\|=M \tau\left(e_{23}-e_{32}\right)$ and $\left\|v_{12}\right\|=$ $M \tau\left(e_{12}-e_{21}\right)$ which is a priori different to $\left\|v_{13}\right\|-\left\|v_{23}\right\|=M \tau\left(e_{13}+e_{32}-e_{31}-e_{23}\right)$. In the following subsection, it is clearly illustrated by 2D and 3D simulations of three grain junction motion by LS approach.

\subsection{Three grain junction motion: independent velocities}

To test the robustness of the LS approach for modeling the recrystallization with averaging the stored energy per interface, a dimensionless simple configuration was considered in a unit square or a unit cube. Namely, the simulations of a triple junction motion was performed imposing constant velocities for interfaces (see Fig. 5 (a)) which

initially meet at the angles of 120 degrees. One of the three interfaces is imposed to be stationary $\left(v_{12}=0\right)$. For two other interfaces, magnitudes of velocities and migration direction can be varied independently. The configurations considered in the present work are inspired by [33] which offers the possibility of comparing the results of the present simulations with expected interface migrations. 


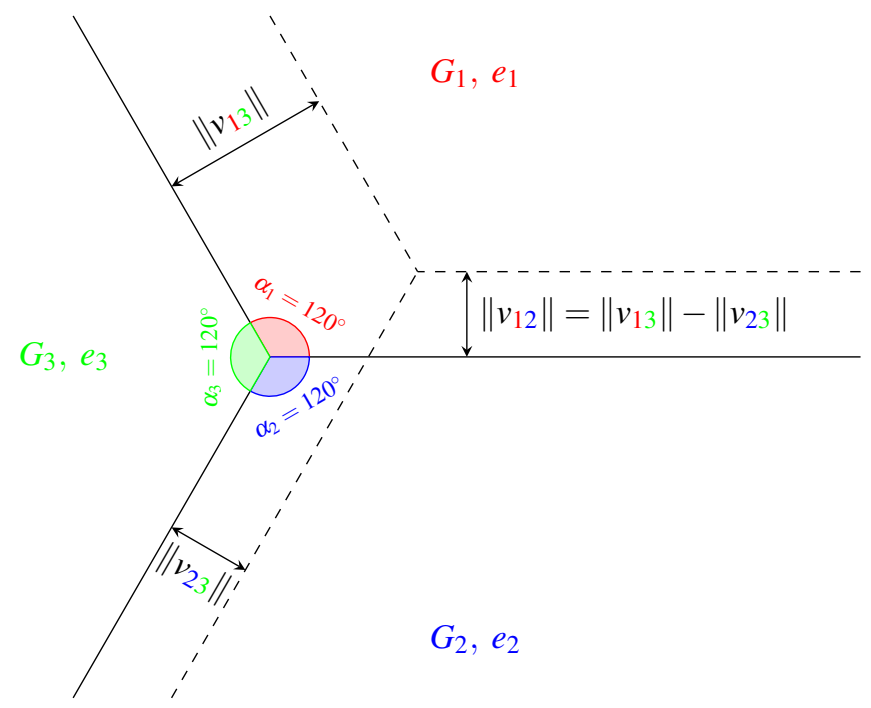

(a)

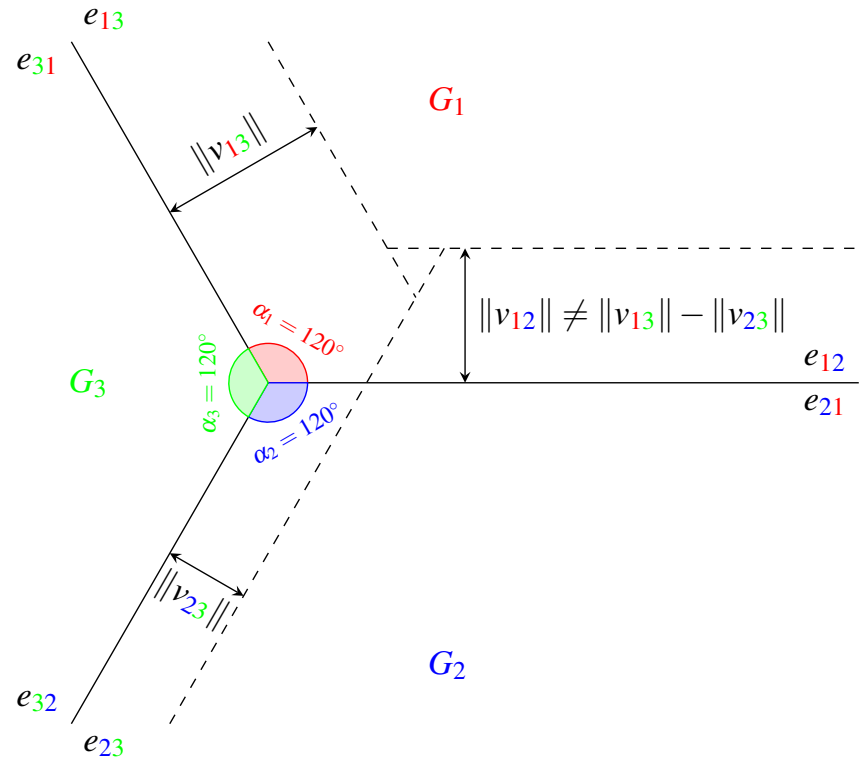

(b)

Figure 4: (a) A triple junction meeting at angles of 120 degrees with compatible displacements in the "per grain" approach. (b) Triple junction with independent boundary velocities and incompatible displacements resulting in curved boundaries in the "per interface" approach. 
In all simulations in the present work, the time step was chosen to provide the displacement of the interfaces at each step to be smaller than the minimal mesh size.

First, convergence of the numerical solution with mesh refinement was verified. Results of 2D simulations with homogeneous isotropic FE mesh are illustrated in Fig. 5 . The numerical solution obtained for the mesh composed of 163,620 elements coincides with the ones provided by simulation with 665,706 elements (Fig. 5 (d)). The FE mesh of 163,620 elements was further used to study other 2D configurations which are illustrated in Fig. 6 All the 2D simulation results are similar to solutions proposed in [33] for the 2D cases. However, capillarity was not taken into account in [33]. This explains that the triple junction can, very locally, differ and the observed smoothed angle in the numerical solution visible in Fig. 6(e) (see the red circle).

For 3D simulations, an isotropic FE mesh containing 14,211,592 tetrahedra was generated for the unit cubic domain with mesh refinement in the vicinity of the interfaces (see Fig. 7). In the case of the interface velocities of the same magnitude, the triple junction motion results in the formation of a spiral type junction. Initial and final (at time $t=80$ ) configurations are shown in Fig. 7. Other triple junction motion problems were considered as it was done for the $2 \mathrm{D}$ case. The simulations results are illustrated in Fig. 8 and conclusions, in terms of convergence and representativeness of the results are similar to ones obtained in 2D simulations.

\section{Static recrystallization simulations}

Simulations of recrystallization for 304L stainless steel were performed with the results of crystal plasticity FE computations adopted from [20] and considering necklacetype nucleation or bulk-type nucleation. The same polycrystal generated by LaguerreVoronoï Tessellation method [37, 38] was considered. Using crystal plasticity analysis, a planar compression test up to a total strain of $30 \%$ was simulated on a polycrystal composed of 100 grains with initial dimensions $0.62 \times 0.62 \times 0.62 \mathrm{~mm}^{3}$. The reader is invited to [20] for details.

After deformation, the dimensions of the polycrystalline aggregate are $0.8 \times 0.62 \times$ $0.48 \mathrm{~mm}^{3}$. A homogeneous isotropic FE mesh composed of 7,683,541 elements was 


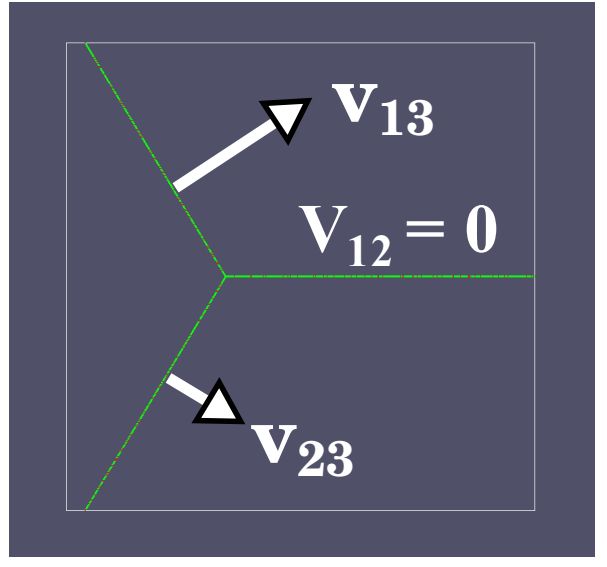

(a)

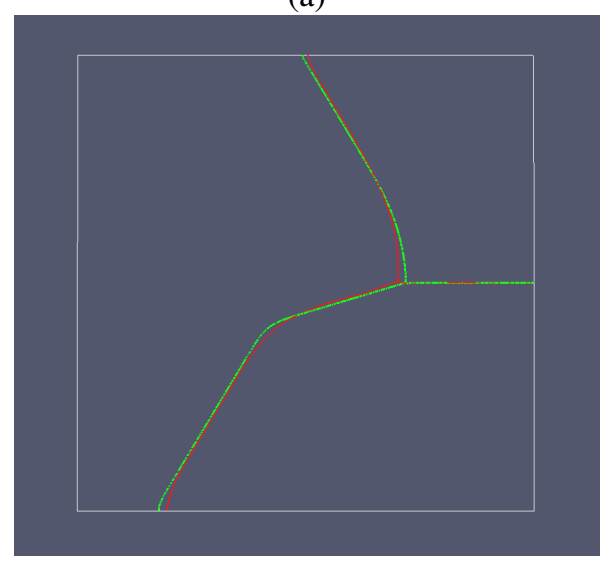

(c)

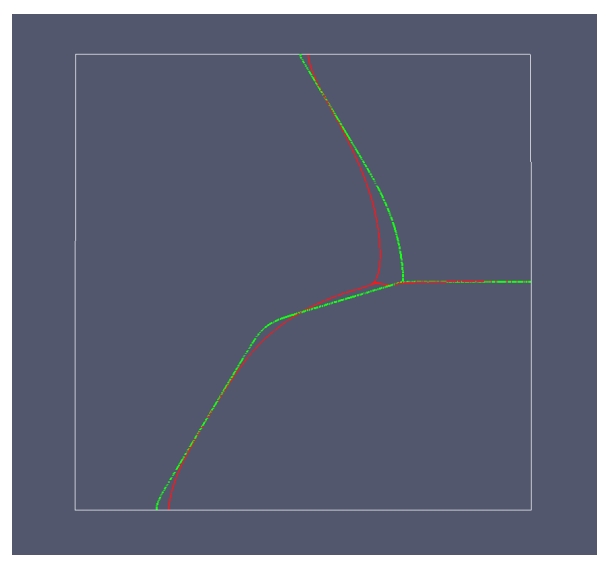

(b)

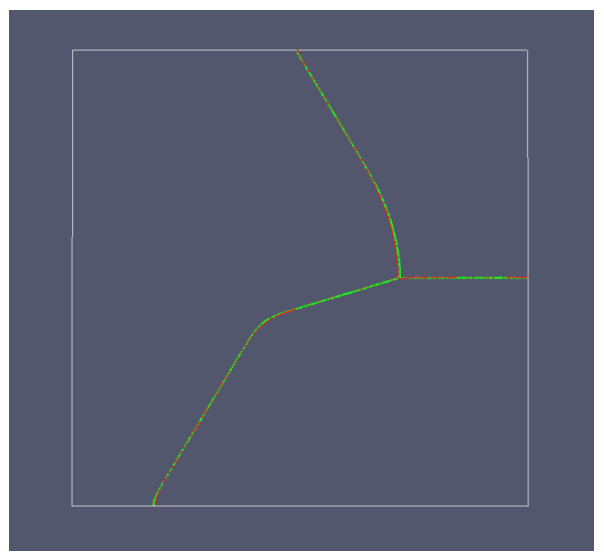

(d)

Figure 5: (a) Three grain junction with initial angles of 120 degrees, and comparison of the results of 2D simulations obtain using isotropic homogeneous FE mesh composed of (b) 12,478 (red curve) and 665,706 (green curve) elements, (c) 40,522 (red curve) and 665,706 (green curve) elements, (d) 163,620 (red curve) and 665,706 (green curve) elements. 


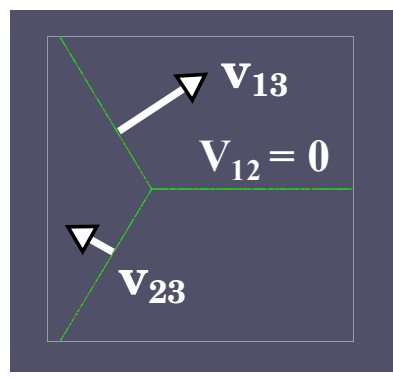

(a)

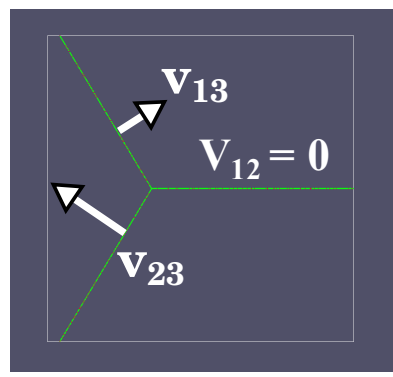

(d)

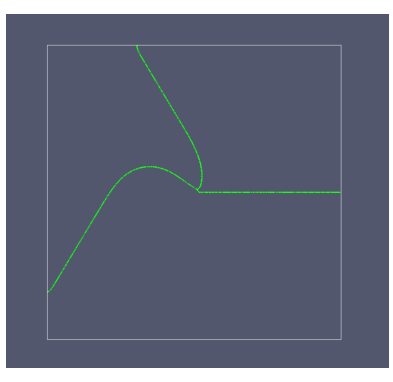

(b)
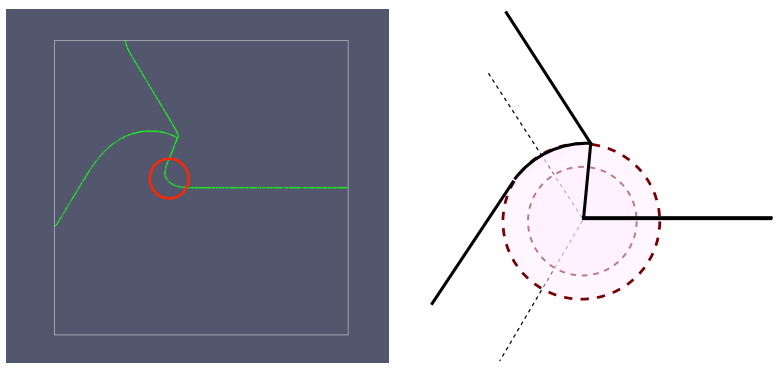

(f)

(e)

Figure 6: Three grain junction with initial angles of 120 degrees with imposed constant velocities (a) $v_{13}>$ $v_{23}, v_{12}=0$, (b) $v_{13}<v_{23}, v_{12}=0$ and (b), (e) corresponding results of 2D simulations; (c), (f) corresponding expected evolutions according to [33] without curvature driven migration.
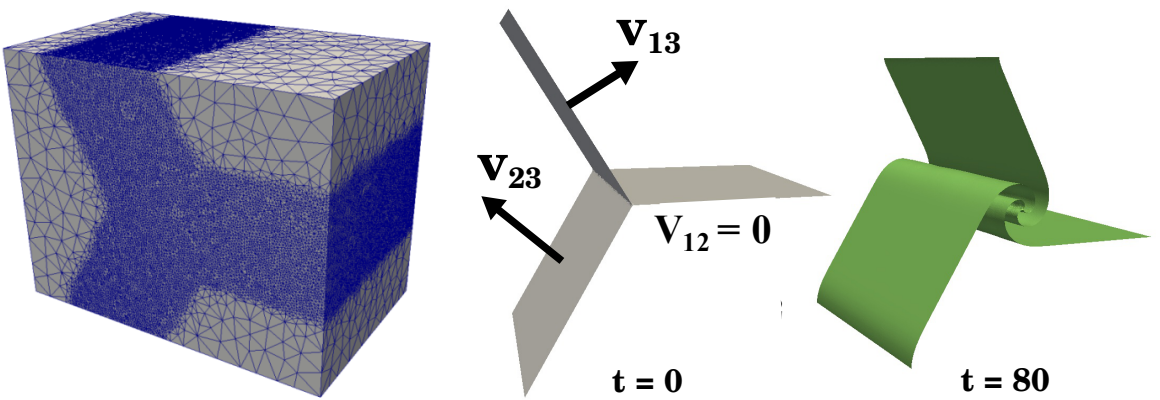

Figure 7: FE mesh used for 3D simulations of three grain junction motion by the LS method with imposed three constant velocities of the same magnitude resulting in the formation of a spiral type junction. 


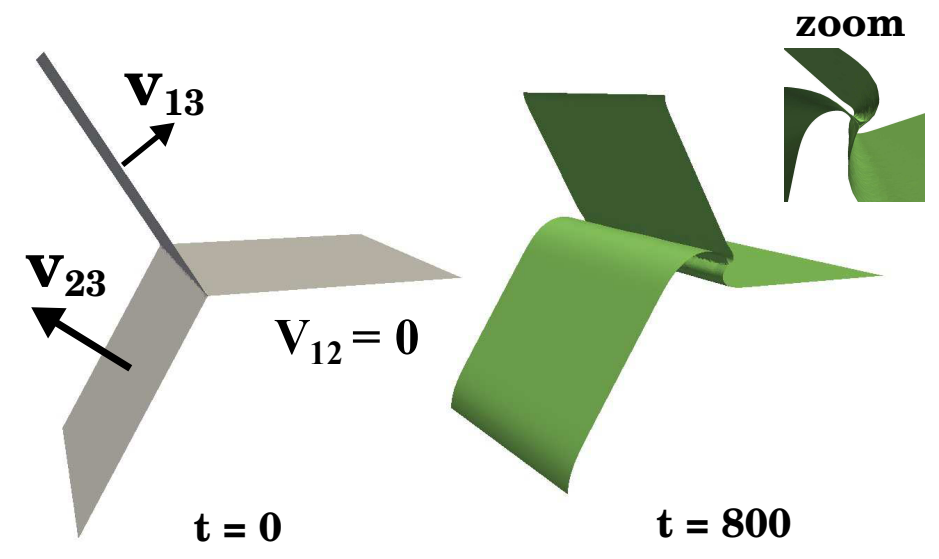

(a)
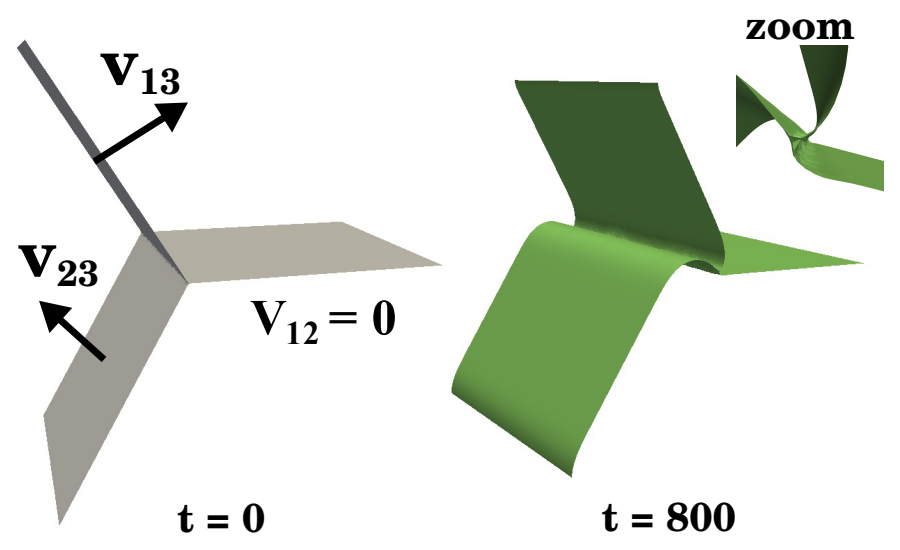

(b)

Figure 8: Three grain junction with initial angles of 120 degrees with imposed constant velocities and results of 3D simulations of junction motion by the LS method (similar to the 2D case shown in Fig. 6 . 
generated for the static recrystallization calculations. A heat treatment of 20 minutes at temperature of $1000^{\circ} \mathrm{C}$ was simulated with time step $\Delta t=5 \mathrm{~s}$. Fig. 9. (a) displays the stored energy field provided by the crystal plasticity computations with added nuclei at GB. Fig. 9 b) illustrates the corresponding stored energy averaged per interface. One can see that stored energy far from the grain interfaces $\left(\phi_{i}>h\right)$ is, as detailed previously in the "per interface" numerical framework, not taken into account in the velocity calculation (blue color in the grains's bulk). Moreover, the energy field seems to fluctuate in the initial unrecrystallized grain interfaces. These fluctuations are consistent with the fact that nucleus are taken into account as new grains to evaluate the per interface stored energy field.

Simulation parameters used in the recrystallization computations are collected in Table 1

\begin{tabular}{|ll|}
\hline Parameter & Value \\
\hline GB mobility, $M$ & $5 \cdot 10^{-13} \mathrm{~m}^{4} \cdot \mathrm{J}^{-1} \cdot \mathrm{s}^{-1}$ \\
GB energy, $\gamma$ & $0.6 \mathrm{~J} \cdot \mathrm{m}^{-2}$ \\
Temperature, $T$ & $1000^{\circ} \mathrm{C}$ \\
Time step, $\Delta t$ & $5 \mathrm{~s}$ \\
Number of nuclei (necklace), $\Upsilon_{g b}$ & 3235 \\
Number of nuclei (bulk), $\Upsilon_{b u l k}$ & 3224 \\
Minimal stored energy, $e_{0}$ & $0 \mathrm{~J} \cdot \mathrm{m}^{-3}$ \\
Thickness of the interface zone, $h$ & $0.03 \mathrm{~mm}$ \\
\hline
\end{tabular}

Table 1: Parameters used in the static recrystallization simulations for the considered 304L steel [20].

The effect of the interface zone thickness, $h$, which is a parameter imposed by the user, need to be discussed. It is worth mentioning that $h$ has to be less or equal to the half-value thickness of the reinitialization zone (where the metric property $\|\phi(x, t)\|=$ 1 is ensured) since the distance function and the extended index field need to be defined for the interface identification as it is described in Sec. 2.3. In addition, it should be higher than a maximal displacement of GBs at any time step. In the simulations per- 


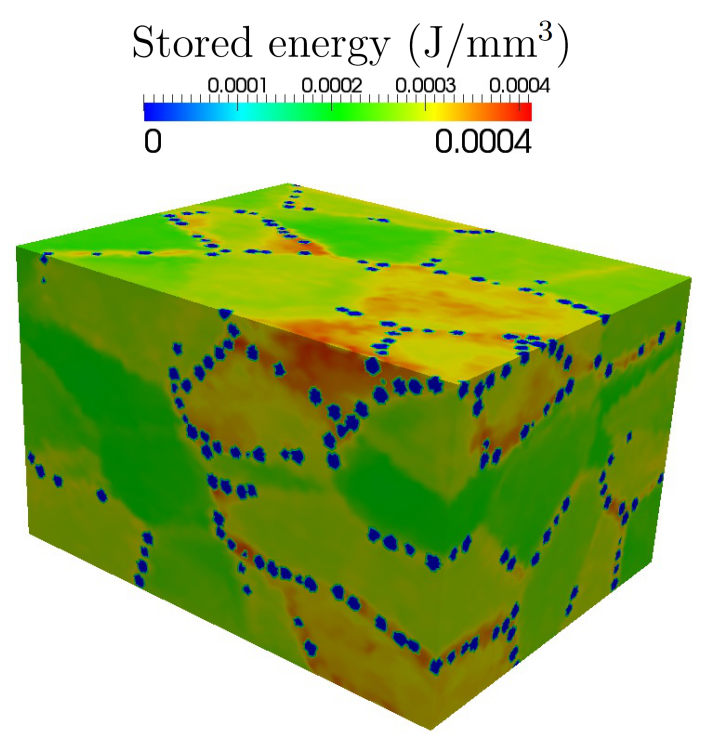

(a)

Stored energy averaged per interface

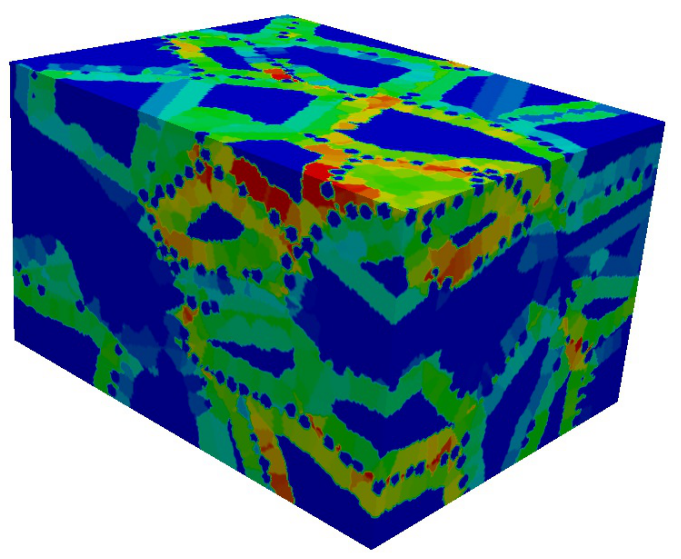

(b)

Figure 9: (a) Stored energy field as provided by the crystal plasticity FE computations (nuclei are shown in blue) and (b) stored energy averaged per interface zones at time $t=0 \mathrm{~s}$. 
formed in the present work, the time step was chosen to ensure that any displacement of GBs for each time increment is less than the mesh size. To perform the simulations with acceptable computation time, $h$ was chosen to be equal to 4 times the mesh size, which is the same as the half-value thickness of the reinitialization zone.

The evolution of recrystallization fraction in the performed simulations are presented in Fig. 10 in comparison with the results reported in [20] for the necklace-type nucleation and bulk-type nucleation. One can see that the recrystallization kinetics differ at the beginning of the heat treatment: a higher recrystallization rate was recorded in the case of "per interface" approach. This results is expectable since the dislocations accumulate mostly in the vicinity of GBs and this should yield a higher GBs migration rate when the GBs passes these zones. In contrast to the "per interface" model, the approach with averaging the energy per grain suppresses this physical aspect. However, the curves obtained using these two methods are close and match at the final stage of the heat treatment.

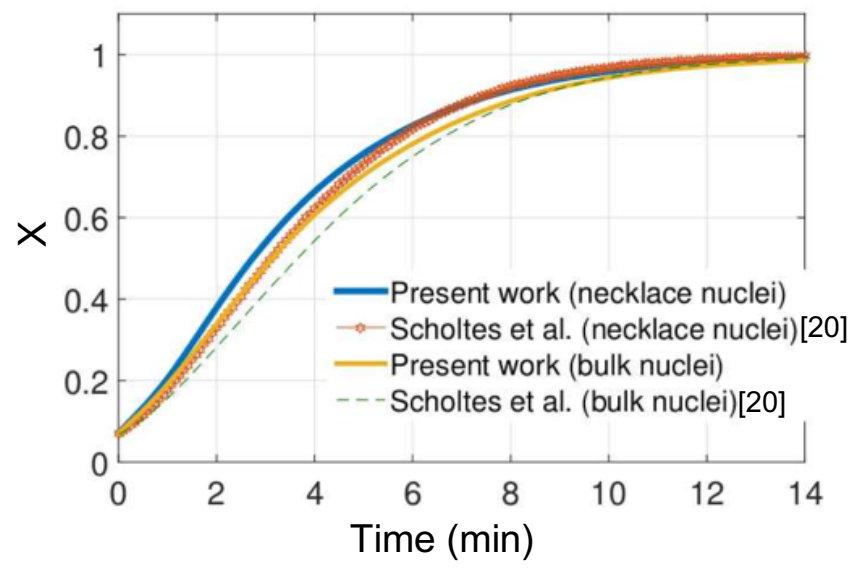

Figure 10: Temporal evolution of the recrystallized volume fraction obtained using the "per interface" (present work) and "per grain" (Scholtes et al. [20]) approaches in cases of necklace type and bulk nucleation.

All the results analyzed further in this subsection were obtained for the necklacetype nucleation and bulk-type nucleation. To compare the models with averaging per 
grain and per interface, the evolution of the mean grain size was plotted. As one can see in Fig. 11, it is greater in the polycrystal issued from the "per interface" simulation.

For the comparison of the grain topology, the analysis of grain volume - grain surface area scattering was performed. The initial scattering as well as the grain size distribution are plotted in Fig. 12 The grain size distributions at time $t=500 \mathrm{~s}$ and $t=1000 \mathrm{~s}$ are shown Fig. 13 a-b) and differ slightly for the two approaches. The distributions are shifted towards higher equivalent grain size radii in the case of "per interface" method. This is in agreement with the data obtained for the mean grain size shown in Fig. 11. The grain volume - area scattering at time $t=500 \mathrm{~s}$ and $t=1000 \mathrm{~s}$ obtained in the simulations using "per grain" and "per interface" averaging can be compared in Fig. 13. (c-f). Higher values of grain surface area were obtained in the case of the "per interface" method as it can be noticed from the comparison of Fig. 13. (c) with Fig. 13(e) and Fig. 13.d) with Fig. 13 f), while the grain volume range is the same for both approaches. These results allow to conclude that the grains issued from the "per interface" simulations dispose more complex topology. This can be seen in Fig. 14 illustrating the grains and middle cross sections of the polycrystal. It is worth mentioning that curved GBs were formed even without strain energy heterogeneity along the interfaces (see, for example, the red circles in Fig. 14(d)). In Fig. 14(c-f), in some places, a debonding phenomenon seems appear between grain interfaces (see for example the green circles in Fig. 14ff)). This effect is purely due to the post-processing where, to limit the storage data, a unique $\varepsilon$-isovalue (with $\varepsilon$ positive and close to zero) of the $\max _{i}\left(\phi_{i}\right)$ is observed in 2D-cuts rather than to exhibit the zero-isovalues of all the GLS functions.

The 3D simulations were realized on 18 Intel Xeon CPUs. Calculation time of the "per interface" simulation was slightly higher (37.5h) than the "per grain" method (35.8h). This difference can be explained by a higher computational cost of the direct reinitialization algorithm in the "per interface" simulations since higher grain surface area is formed as it was shown before. 


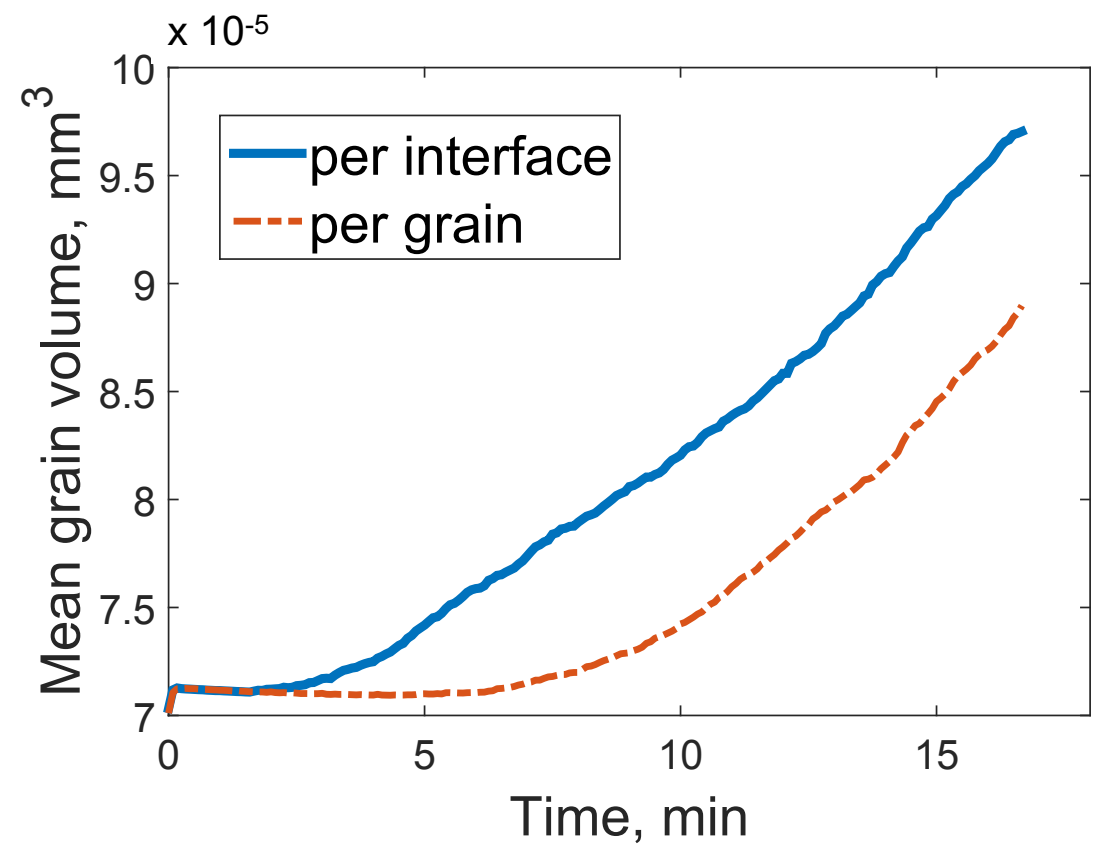

Figure 11: Temporal evolution of the mean grain volume for the polycrystal with necklace-type nucleation obtained in the simulations with averaging the stored energy per grain [20] and per interface.

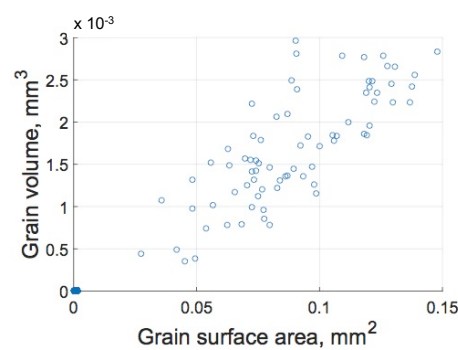

(a) Initial

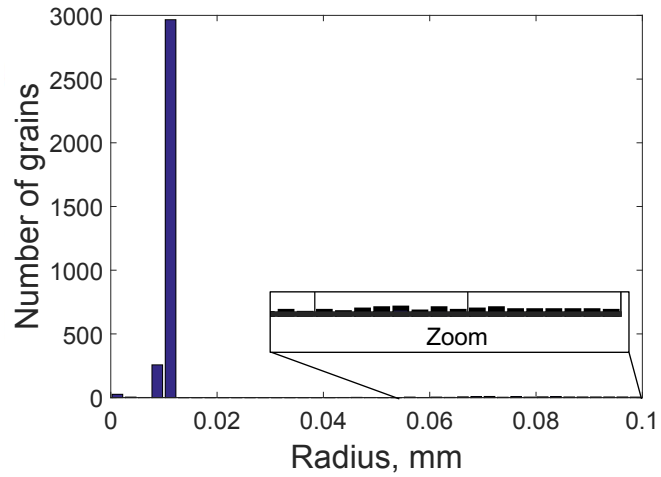

(b) Initial

Figure 12: (a) Initial grain surface area - grain volume scattering and (b) grain size distribution for the simulation with necklace-type nucleation. 


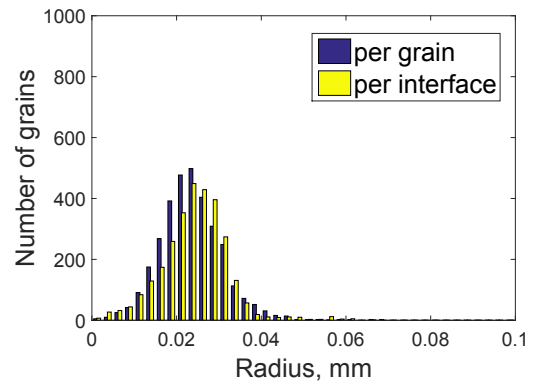

(a) $t=500 \mathrm{~s}$

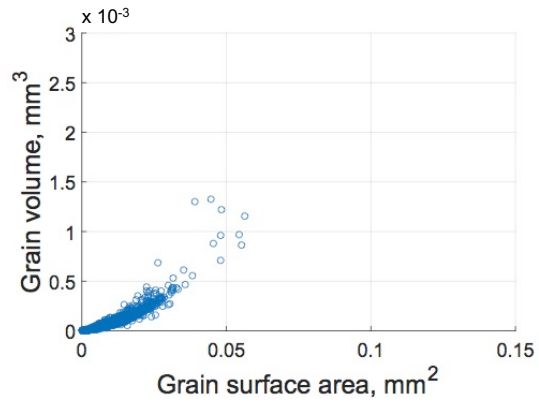

(c) Per grain, $t=500 \mathrm{~s}$

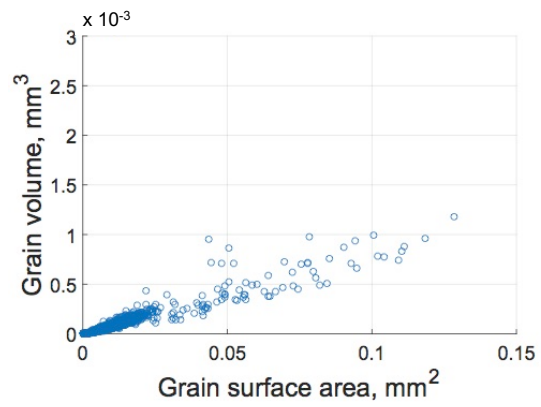

(e) Per interface, $t=500 \mathrm{~s}$

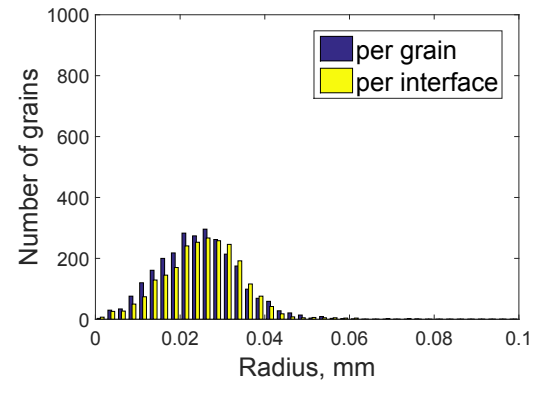

(b) $t=1000 \mathrm{~s}$

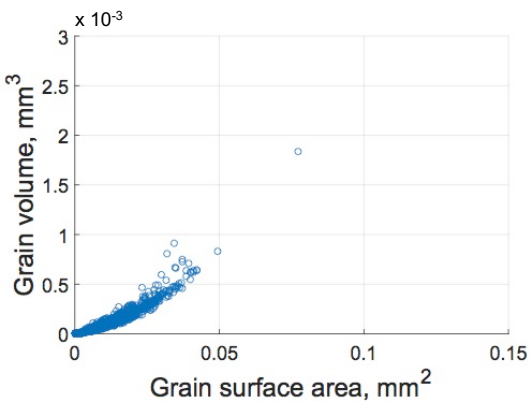

(d) Per grain, $t=1000 \mathrm{~s}$

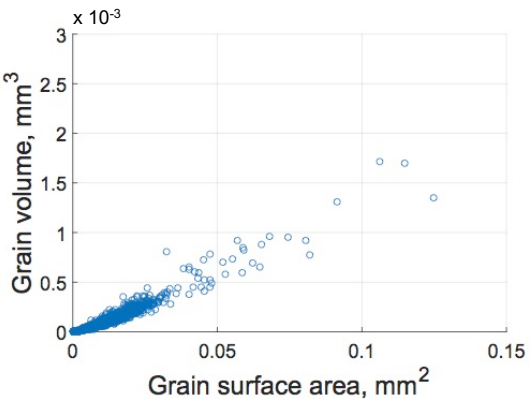

(f) Per interface, $t=1000 \mathrm{~s}$

Figure 13: (a) Comparison of grain size distributions at time $t=500 \mathrm{~s}$ and (b) $t=1000 \mathrm{~s}$. (c) Grain surface area - grain volume scattering as provided by "per grain" approach at time $t=500 \mathrm{~s}$ and (d) $t=1000 \mathrm{~s}$, (e) area - volume scattering as provided by "per interface" method at time $t=500 \mathrm{~s}$ and (f) $t=1000 \mathrm{~s}$. 


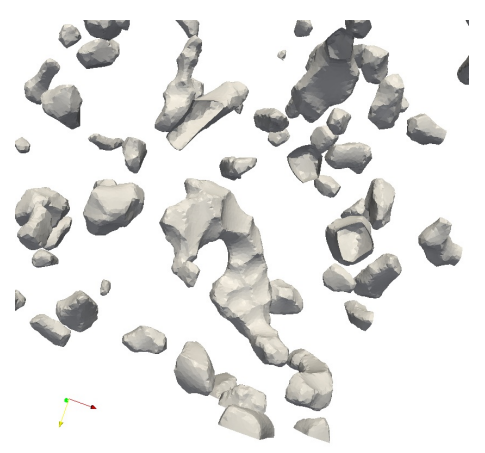

(a)

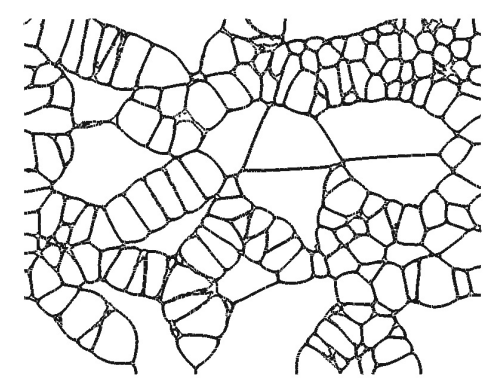

(c) per grain, $t=250 \mathrm{~s}$

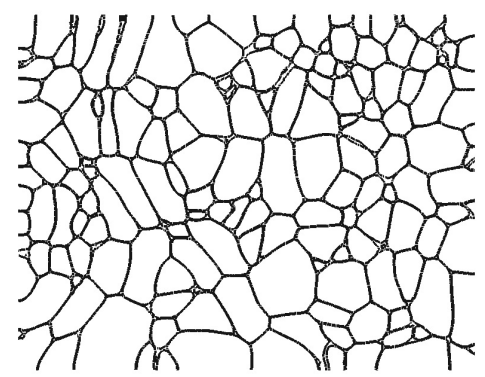

(e) per grain, $t=1000 \mathrm{~s}$

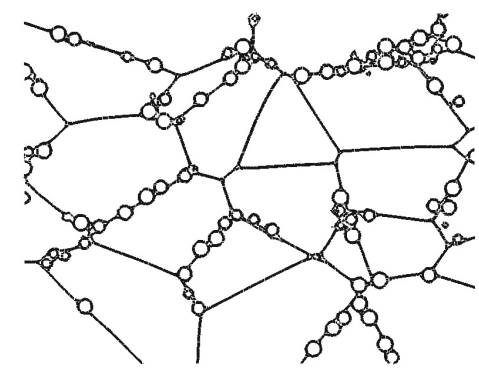

(b)

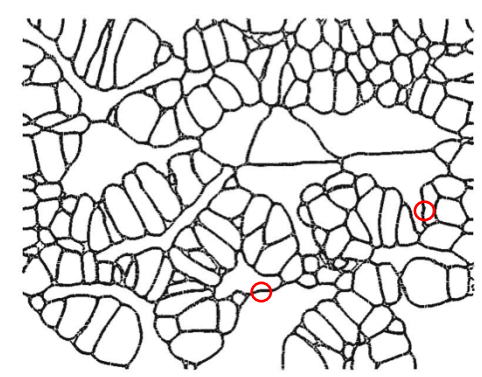

(d) per interface, $t=250 \mathrm{~s}$

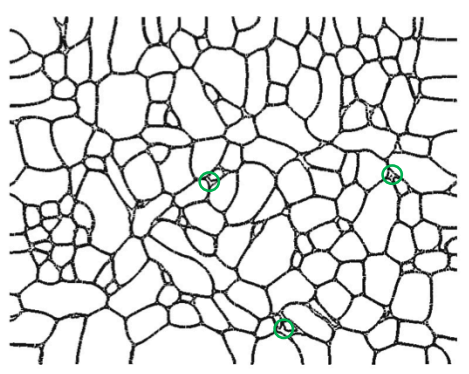

(f) per interface, $t=1000 \mathrm{~s}$

Figure 14: (a) Grains of one of the GLS at time $t=1000 s$ obtained in the simulation with averaging per interface. (b) Middle cross section of the grain structure at the initial state, (c) middle section obtained in the simulation using "per grain" and (d) "per interface" methods at time $t=250 \mathrm{~s}$ (red circles correspond to example of curved GBs); (e) middle section obtained using "per grain" and (f) "per interface" methods at time $t=1000 \mathrm{~s}$ (green circles correspond to example of post-processing artifacts). 


\section{Effect of heterogeneities along GB}

To study the effect of the stored energy heterogeneity along GB, a small system containing 12 grains and 1 nucleus with dimensions $0.2 \times 0.155 \times 0.12 \mathrm{~mm}^{3}$ was extracted from the polycrystal considered in the previous subsection. An isotropic homogeneous FE mesh composed of 7,683,541 elements was used in the simulation. The stored energy field was interpolated from the original coarse mesh thereby smoothing it along GB. The time step $\Delta t=1 s$ was chosen to ensure that the displacement of a migrating GBs at each step is less than the mesh size.

Fig. 15 illustrates the grain structure of the extracted part with the corresponding stored energy field. The nucleus can be clearly distinguished in Fig. 15 by the blue color which corresponds to the minimal strain energy $e_{0}=0$.

In the vicinity of multiple junctions, the velocity field, $\vec{v}_{e}$, was smoothed as it was originally proposed in [17] instead of using Eq. [14]:

$$
\vec{v}_{e}(x, t)=M \sum_{i=1}^{N_{p}} \sum_{j=1, j \neq i}^{N_{p}} \chi_{i}(x, t) f\left(\phi_{i}(x, t), l\right)\left(\varepsilon_{i}(x, t)-\varepsilon_{j}(x, t)\right) \vec{n}_{i j}(x, t),
$$

where $\chi_{i}$ is the characteristic function for the grain family $i$ and $\varepsilon_{i}(x, t)$ is the stored energy field (without averaging).

To compute the energy difference across the interface, $\varepsilon_{i}(x, t)-\varepsilon_{j}(x, t)$, the reinitialization/extension procedure was used as it was implemented in [20]. Namely, the global stored energy field was decomposed on $N_{p}$ fields according to the grain families. Each field is equal to the original energy value in nodes where $\phi_{i}(x, t) \geq 0$ and -1 otherwise. Next, the extensions for the energy fields are constructed: the value from each node which is the closest to a surface element of the interface of $\phi_{i}$ (discretized during the reinitialization procedure) but not in $\phi_{i}$ is transmitted across the interface to nodes in $\phi_{i}$ closer to this surface element of the interface than any other, up to the thickness $h$. This can be done thanks to the $k-d$ tree constructed in the reinitialization algorithm [20].

The interfaces obtained for the time $t=250 \mathrm{~s}$ in the simulations without averaging procedure are shown in comparison with the results of computation using the "per interface" method. The shapes of the GBs at the transient state obtained in the simulations 
without averaging (Fig. 15.e)) are much more complex compared to the ones provided by the approach with interface treatment (Fig. $15(\mathrm{c}))$. The resulting microstructures at time $t=1000 \mathrm{~s}$ for these approaches differ significantly as one can see in Fig. 15f) and Fig.15(d).

These results demonstrate clearly that the strain energy heterogeneities along GB, affect both the transient and final grain shapes and size in the microstructure under thermomechanical processing. However, due to the very high computational cost of the simulations without averaging procedure, only small systems have been studied at yet.

\section{Conclusions}

The summary of the present work is the following. The GBs motion driven by stored energy gradient in polycrystalline microstructure was reconsidered by treating the energy heterogeneities at the intragranular scale. First, the stored energy in the grain was averaged over the adjacent zone of each GBs and not over the whole grain as in previous deterministic full field works. This treatment provides more accurate simulation from the physical point of view with low supplementary computational cost.

In contrast to the approach which averages the stored energy per grain and provides the regular shape of GBs in the simulations of static recrystallization, the proposed per interface method demonstrates that GBs distortions can be produced. This was revealed in the analysis of three GBs junction motion with prescribed constant GBs velocities. The obtained simulation results are in good agreement with solutions found in the literature.

For the large SRX simulations, a higher recrystallization rate at the early stage of heat treatment was observed in comparison with the results of simulations in which the stored energy was averaged per grain. The analysis of grain volume and grain surface area scattering shows that in the simulations using "per interface" method the distribution of corresponding points is shifted towards higher values of surface area compared to the simulations using "per grain" method. This is in agreement with the results obtained for the three grain junction motion. The results of small system 


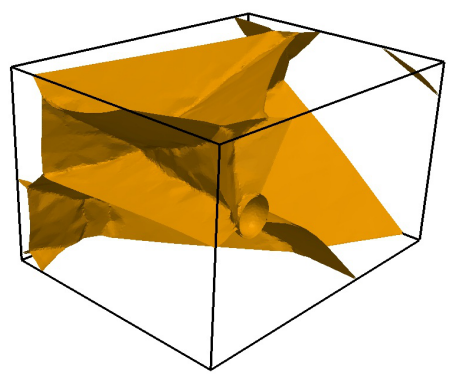

(a) $t=0 \mathrm{~s}$

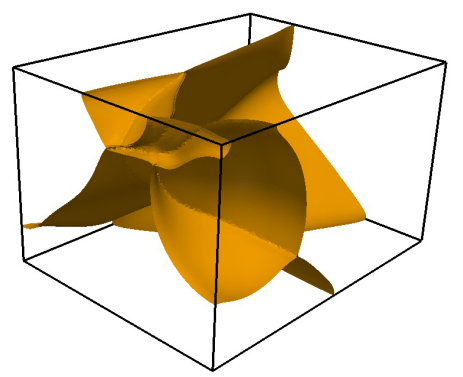

(c) Per interface, $t=250 \mathrm{~s}$

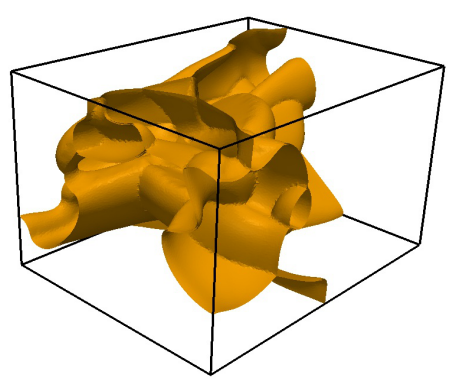

(e) No averaging, $t=250 \mathrm{~s}$

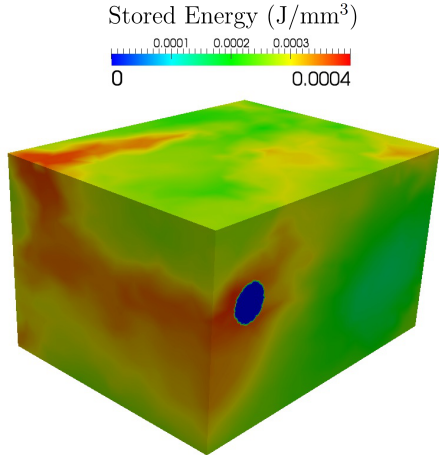

(b) $t=0 \mathrm{~s}$

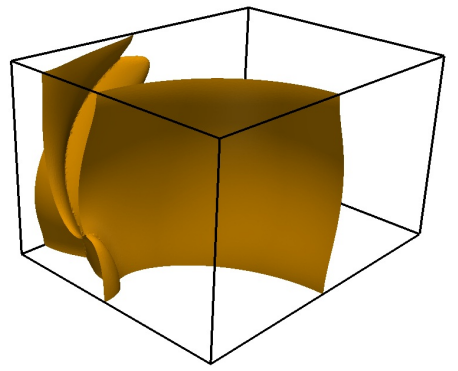

(d) Per interface, $t=1000 \mathrm{~s}$

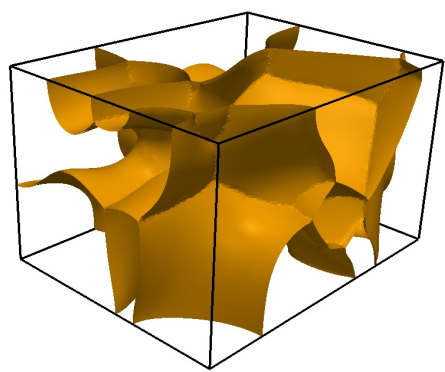

(f) No averaging, $t=1000 \mathrm{~s}$

Figure 15: Comparison of the static recrystallization in (a) the polycrystal with 12 grains and 1 nucleus extracted from the data provided by CPFEM with (b) the corresponding stored energy field. (c) Results obtained by "per interface" method at time $t=250 \mathrm{~s}$ and (d) $t=1000 \mathrm{~s}$, and (e) without averaging at time $t=250 \mathrm{~s}$ and (f) $t=1000 \mathrm{~s}$. 
simulations with few grains and fine mesh revealed that even more distorted GBs can develop if one considers strain heterogeneities along GB.

The findings of the present work are supported by experimental observations of static recrystallization were tortuosity of grains are always more pronounced than during pure grain growth regime. Moreover the method described here will be further used for developing a new LS model to simulate the formation of annealing twins, which has been shown to occur preferentially along with the migration of the recrystallized front and is likely to be promoted by the tortuosity of this interface [19, 39].

\section{Acknowledgements}

This work has been funded and carried out within the frame of the OPALE industrial chair co-funded by Safran and the French National Agency for Research ANR (grant number ANR-14-CHIN-0002-01).

\section{References}

[1] R. Doherty, D. Hughes, F. Humphreys, J. Jonas, D. Jensen, M. Kassner, W. King, T. McNelley, H. McQueen, A. Rollett, Current issues in recrystallization: a review, Materials Science and Engineering: A 238 (2) (1997) 219 - 274. doi:https://doi.org/10.1016/S0921-5093(97)00424-3

URL http://www.sciencedirect.com/science/article/pii/ S0921509397004243

[2] A. Rollett, D. Srolovitz, R. Doherty, M. Anderson, Computer simulation of recrystallization in non-uniformly deformed metals, Acta Metallurgica 37 (2) (1989) 627 - 639. doi:https://doi.org/10.1016/0001-6160(89) 90247-2.

URL http://www.sciencedirect.com/science/article/pii/ 0001616089902472

[3] B. Radhakrishnan, G. Sarma, T. Zacharia, Modeling the kinetics and microstructural evolution during static recrystallizationmonte carlo simulation of recrystallization Acta Materialia 46 (12) (1998) 4415 - 4433. 
doi:https://doi.org/10.1016/S1359-6454(98)00077-9

URL http://www.sciencedirect.com/science/article/pii/ S1359645498000779

[4] B. Radhakrishnan, G. Sarma, T. Zacharia, Monte carlo simulation of deformation substructure evolution during recrystallization, Scripta Materialia 39 (12) (1998) $1639-1645$.

[5] L. Zhang, A. D. Rollett, T. Bartel, D. Wu, M. T. Lusk, A calibrated monte carlo approach to quantify the impacts of misorientation and different driving forces on texture development, Acta Materialia 60 (3) (2012) 1201 - 1210. doi:https://doi.org/10.1016/j.actamat.2011.10.057.

URL http://www.sciencedirect.com/science/article/pii/ S1359645411007725

[6] W. Wang, A. Helbert, F. Brisset, M. Mathon, T. Baudin, Monte carlo simulation of primary recrystallization and annealing twinning, Acta Materialia 81 (Supplement C) (2014) 457 - 468. doi:https: //doi.org/10.1016/j.actamat.2014.08.032 URL http://www.sciencedirect.com/science/article/pii/ S1359645414006338

[7] D. Raabe, Cellular Automata in Materials Science with Particular Reference to Recrystallization Simulation, Annual Review of Materials Research 32 (1) (2002) 53-76. doi:10.1146/annurev.matsci.32.090601.152855

URL http://www.annualreviews.org/doi/10.1146/annurev.matsci. 32.090601 .152855

[8] K. Janssens, An introductory review of cellular automata modeling of moving grain boundaries in polycrystalline materials, Mathematics and Computers in Simulation 80 (7) (2010) 1361 - 1381, multiscale modeling of moving interfaces in materials. doi:https://doi.org/10.1016/j.matcom.2009.02.011.

URL http://www.sciencedirect.com/science/article/pii/ S0378475409000470 
[9] L. Sieradzki, L. Madej, A perceptive comparison of the cellular automata and monte carlo techniques in application to static recrystallization modeling in polycrystalline materials Computational Materials Science 67 (Supplement C) (2013) 156 - 173. doi:https://doi.org/10.1016/j.commatsci.2012.08.047 URL http://www.sciencedirect.com/science/article/pii/ S0927025612005435

[10] H. Garcke, B. Nestler, B. Stoth, A multiphase field concept: Numerical simulations of moving phase boundaries and multiple junctions, SIAM Journal on Applied Mathematics 60 (1) (1999) 295-315.

[11] N. Moelans, B. Blanpain, P. Wollants, A phase field model for the simulation of grain growth in materials containing finely dispersed incoherent second-phase particles, Acta Materialia 53 (6) (2005) 1771 - 1781. doi:https://doi.org/10.1016/j.actamat.2004.12.026

URL http://www.sciencedirect.com/science/article/pii/ S1359645404007621

[12] S. Esedolu, Grain size distribution under simultaneous grain boundary migration and grain rotation in two dimensions, Computational Materials Science 121 (2016) 209 - 216. doi:https://doi.org/10.1016/j.commatsci.2016. 04.022

URL http://www.sciencedirect.com/science/article/pii/ S0927025616301811

[13] A. Harun, E. A. Holm, M. P. Clode, M. A. Miodownik, On computer simulation methods to model zener pinning, Acta Materialia 54 (12) (2006) 3261 - 3273. doi:https://doi.org/10.1016/j.actamat.2006.03.012. URL http://www.sciencedirect.com/science/article/pii/ S135964540600214X

[14] G. Couturier, C. Maurice, R. Fortunier, Three-dimensional finite-element simulation of zener pinning dynamics, Philosophical Magazine 83 (30) (2003) 33873405. 
[15] S. Osher, J. A. Sethian, Fronts propagating with curvature-dependent speed: Algorithms based on hamilton-jacobi formulations, Journal of Computational Physics 79 (1) (1988) 12 - 49. doi:https://doi.org/10.1016/ 0021-9991(88)90002-2.

URL http://wwW.sciencedirect.com/science/article/pii/ 0021999188900022

[16] M. Bernacki, Y. Chastel, H. Digonnet, H. Resk, T. Coupez, R. Log, Development of numerical tools for the multiscale modelling of recrystallization in metals, based on a digital material framework, Computer Methods in Materials Science Vol. 7, No. 1 (2007) 142-149.

[17] M. Bernacki, Y. Chastel, T. Coupez, R. Logé, Level set framework for the numerical modelling of primary recrystallization in polycrystalline materials, Scripta Materialia 58 (12) (2008) 1129-1132. doi:10.1016/j.scriptamat.2008.02.016

URL http://linkinghub.elsevier.com/retrieve/pii/ S1359646208001425

[18] M. Bernacki, R. Logé, T. Coupez, Level set framework for the finiteelement modelling of recrystallization and grain growth in polycrystalline materials, Scripta Materialia 64 (6) (2011) 525-528. doi: $10.1016 / \mathrm{j}$.scriptamat.2010.11.032

URL http://linkinghub.elsevier.com/retrieve/pii/ S1359646210007906

[19] Y. Jin, B. Lin, A. D. Rollett, G. S. Rohrer, M. Bernacki, N. Bozzolo, Thermomechanical factors influencing annealing twin development in nickel during recrystallization. Journal of Materials Science 50 (15) (2015) 5191-5203. doi: 10.1007/s10853-015-9067-0.

URL https://doi.org/10.1007/s10853-015-9067-0

[20] B. Scholtes, R. Boulais-Sinou, A. Settefrati, D. Pino Muñoz, I. Poitrault, A. Montouchet, N. Bozzolo, M. Bernacki, 3D level set modeling of static 
recrystallization considering stored energy fields, Computational Materials Science 122 (2016) 57-71.

URL http://linkinghub.elsevier.com/retrieve/pii/ S0927025616302142

[21] L. Maire, B. Scholtes, C. Moussa, N. Bozzolo, D. Pino Muñoz, A. Settefrati, M. Bernacki, Modeling of dynamic and post-dynamic recrystallization by coupling a full field approach to phenomenological laws, Materials Design 133 (Supplement C) (2017) 498 - 519. doi:https://doi.org/10.1016/j.matdes.2017.08.015

URL http://www.sciencedirect.com/science/article/pii/ S026412751730761X

[22] H. Hallberg, A modified level set approach to $2 \mathrm{~d}$ modeling of dynamic recrystallization, Modelling and Simulation in Materials Science and Engineering 21 (8) (2013) 085012.

URL http://stacks . iop.org/0965-0393/21/i=8/a=085012

[23] M. Elsey, S. Esedoglu, P. Smereka, Diffusion generated motion for grain growth in two and three dimensions, Journal of Computational Physics 228 (21) (2009) 8015 - 8033. doi:https://doi.org/10.1016/j.jcp.2009.07.020

URL http://www.sciencedirect.com/science/article/pii/ S0021999109004082

[24] M. Elsey, S. Esedolu, P. Smereka, Large-scale simulation of normal grain growth via diffusion-generated motion, Proceedings of the Royal Society of London A: Mathematical, Physical and Engineering Sciences 467 (2126) (2010) 381-401. arXiv:http://rspa.royalsocietypublishing.org/content/ 467/2126/381.full.pdf. doi:10.1098/rspa.2010.0194.

URL http://rspa.royalsocietypublishing.org/content/467/2126/ 381

[25] C. Mießen, M. Liesenjohann, L. Barrales-Mora, L. Shvindlerman, G. Gottstein, An advanced level set approach to grain growth accounting for grain boundary 
anisotropy and finite triple junction mobility, Acta Materialia 99 (Supplement C)

(2015) 39 - 48. doi:https://doi.org/10.1016/j.actamat.2015.07.040.

URL http://www.sciencedirect.com/science/article/pii/

S135964541500511X

[26] M. Bernacki, H. Resk, T. Coupez, R. E. Logé, Finite element model of primary recrystallization in polycrystalline aggregates using a level set framework, Modelling and Simulation in Materials Science and Engineering 17 (6) (2009) 064006. URL http://stacks.iop.org/0965-0393/17/i=6/a=064006

[27] J. Humphreys, G. S. Rohrer, A. Rollett, Chapter 16 - Computer Modeling and Simulation of Annealing, third edition Edition, Elsevier, Oxford, 2017. doi : https://doi.org/10.1016/B978-0-08-098235-9.00016-1.

URL http://www.sciencedirect.com/science/article/pii/ B9780080982359000161

[28] M. Shakoor, B. Scholtes, P.-O. Bouchard, M. Bernacki, An efficient and parallel level set reinitialization method application to micromechanics and microstructural evolutions, Applied Mathematical Modelling 39 (23) (2015) 7291 - 7302. doi:https://doi.org/10.1016/j.apm.2015.03.014.

URL http://www.sciencedirect.com/science/article/pii/ S0307904X15001638

[29] B. Scholtes, M. Shakoor, A. Settefrati, P.-O. Bouchard, N. Bozzolo, M. Bernacki, New finite element developments for the full field modeling of microstructural evolutions using the level-set method, Computational Materials Science 109 (Supplement C) (2015) 388 - 398. doi:https://doi.org/10.1016/j.commatsci.2015.07.042

URL http://www.sciencedirect.com/science/article/pii/ S0927025615004528

[30] G. Abrivard, E. Busso, S. Forest, B. Appolaire, Phase field modelling of grain boundary motion driven by curvature and stored energy gradients. part $\mathrm{i}$ : theory 
and numerical implementation, Philosophical Magazine 92 (28-30) (2012) 36183642. doi:10.1080/14786435.2012.713135

[31] Y. Mellbin, H. Hallberg, M. Ristinmaa, A combined crystal plasticity and graph-based vertex model of dynamic recrystallization at large deformations, Modelling and Simulation in Materials Science and Engineering 23 (4) (2015) 045011. doi:10.1088/0965-0393/23/4/045011. URL http://www.scopus.com/inward/record.url?eid=2-s2. 0-84928999862\{\&\}partnerID=tZOtx3y1

[32] R. Logé, M. Bernacki, H. Resk, L. Delannay, H. Digonnet, Y. Chastel, T. Coupez, Linking plastic deformation to recrystallization in metals using digital microstructures. Philosophical Magazine 88 (30-32) (2008) 3691-3712. doi:10.1080/ 14786430802502575 .

URL http://dx.doi.org/10.1080/14786430802502575

[33] F. Reitich, H. M. Soner, Three-phase boundary motions under constant velocities. i: The vanishing surface tension limit, Proceedings of the Royal Society of Edinburgh: Section A Mathematics 126 (4) (1996) 837865. doi:10.1017/ S0308210500023106.

[34] J. Humphreys, G. S. Rohrer, A. Rollett, Chapter 5 - mobility and migration of boundaries, in: J. Humphreys, G. S. Rohrer, A. Rollett (Eds.), Recrystallization and Related Annealing Phenomena (Third Edition), third edition Edition, Elsevier, Oxford, 2017, pp. 145 - 197. doi:https://doi.org/10.1016/B978-0-08-098235-9.00005-7 URL https://wwW.sciencedirect.com/science/article/pii/ B9780080982359000057

[35] B. Merriman, J. K. Bence, S. J. Osher, Motion of Multiple Junctions: A Level Set Approach, Journal of Computational Physics 112 (2) (1994) 334-363. doi:10.1006/jcph.1994.1105

URL http://linkinghub.elsevier.com/retrieve/pii/ S0021999184711053 
[36] J. E. Bailey, P. B. Hirsch, The recrystallization process in some polycrystalline metals, Proceedings of the Royal Society of London A: Mathematical, Physical and Engineering Sciences 267 (1328) (1962) 11-30. arXiv:http:// rspa.royalsocietypublishing.org/content/267/1328/11.full.pdf. doi:10.1098/rspa.1962.0080

URL http://rspa.royalsocietypublishing.org/content/267/1328/ 11

[37] K. Hitti, P. Laure, T. Coupez, L. Silva, M. Bernacki, Precise generation of complex statistical Representative Volume Elements (RVEs) in a finite element context, Computational Materials Science 61 (2012) 224-238.

[38] K. Hitti, M. Bernacki, Optimized dropping and rolling (odr) method for packing of poly-disperse spheres Applied Mathematical Modelling 37 (8) (2013) 5715 5722. doi:https://doi.org/10.1016/j.apm.2012.11.018.

URL http://www.sciencedirect.com/science/article/pii/ S0307904X12007342

[39] Y. Jin, N. Bozzolo, A. Rollett, M. Bernacki, 2d finite element modeling of misorientation dependent anisotropic grain growth in polycrystalline materials: Level set versus multi-phase-field method, Computational Materials Science 104 (Supplement C) (2015) 108 - 123. doi:https://doi.org/10.1016/j.commatsci.2015.03.012

URL http://www.sciencedirect.com/science/article/pii/ S0927025615001743 